ISSN 1855-3966 (printed edn.), ISSN 1855-3974 (electronic edn.)

ARS MATHEMATICA CONTEMPORANEA 19 (2020) 363-374

https://doi.org/10.26493/1855-3974.1851.b44

(Also available at http://amc-journal.eu)

\title{
Frobenius groups which are the automorphism groups of orientably-regular maps*
}

\author{
Hai-Peng Qu \\ School of Mathematics and Computer Science, Shan Xi Normal University, \\ Shan Xi, P.R.C. \\ Yan Wang ${ }^{\dagger}$ (D), Kai Yuan \\ School of Mathematics and Information Science, Yan Tai University, Yan Tai, P.R.C.
}

Received 12 November 2018, accepted 21 September 2020, published online 22 November 2020

\begin{abstract}
In this paper, we prove that a Frobenius group (except for those which are dihedral groups) can only be the automorphism group of an orientably-regular chiral map. The necessary and sufficient conditions for a Frobenius group to be the automorphism group of an orientably-regular chiral map are given. Furthermore, these orientably-regular chiral maps with Frobenius automorphisms are proved to be normal Cayley maps. Frobenius groups conforming to these conditions are also constructed.
\end{abstract}

Keywords: Frobenius group, (orientably) regular map, automorphism group.

Math. Subj. Class. (2020): 05C25, 05C30

\section{Introduction}

Maps are 2-cell embeddings of graphs in compact, connected surfaces. A flag of a map is a topological triangle whose corners are a vertex, the midpoint of an edge incident with the vertex, and the midpoint of a face incident to both the vertex and the edge. Thus, the supporting surface of any map can be decomposed into flags (considered as closed discs bounded by the triangles).

* The authors want to thank Professor Gareth A. Jones of University of Southampton for his valuable suggestions and encouragement. We also thank the referees for their helpful comments.

${ }^{\dagger}$ Corresponding author. Supported by NSFC (No. 11771258, 11471198, 11671347, 61771019, 11671276), NSFS (No. ZR2017MA022, ZR201910270119) and J16LI02.

E-mail addresses: orcawhale@163.com (Hai-Peng Qu), wang_yan@pku.org.cn (Yan Wang), pktide@163.com (Kai Yuan)

()(7) This work is licensed under https://creativecommons.org/licenses/by/4.0/ 
It is well known that the automorphism group of a map acts semi-regularly on its flags. If the automorphism group of a map is regular on the flags, then the map is called regular. Regular maps have the largest automorphism groups, acting regularly on flags of the map. Similarly, orientably-regular maps are maps in orientable surfaces that have the largest orientation preserving automorphism groups acting regularly on darts (edges with direction).

Regular and orientably-regular maps constitute the most meaningful generalization of the Platonic solids. Early recognition of the importance of regular maps in modern mathematics goes back to Kepler [12]; more recent development of the theory of maps was closely related to the theory of map colorings, with the topic of highly symmetric maps always at the center of interest. The study of regular maps is nowadays considered one of the 'classical' areas of mathematics (e.g., Heffter [7], Klein [13], Dyck [5], or Burnside [3]).

A group $G$ acting on a set $X$ is said to act regularly, if for any pair of elements $x, y \in X$ there exists a unique element $g \in G$ mapping $x$ to $y, x^{g}=y$. In such a case, $X$ can be identified with the elements of $G$, and consequently, any mathematical structure with an automorphism group acting regularly on its base set can be identified with the group itself, the building blocks of the structure being identified with cosets of stabilizers of some blocks. This identification has been used in the theory of regular and orientably-regular maps as well and we just sum up the basics, referring for details to [11] and [2] for the theory of maps on orientable and on general surfaces, and to [16] for a recent survey of the theory of regular and orientably-regular maps. In all the forthcoming group presentations we will assume that the listed exponents are the true orders of the corresponding elements.

A finite regular map $\mathcal{M}$ can in this way be identified with a (partial) three-generator presentation of a finite group $G$, isomorphic to the automorphism group $\operatorname{Aut}(\mathcal{M})$ of $\mathcal{M}$, of the form

$$
G=\left\langle x, y, z \mid x^{2}, y^{2}, z^{2},(x y)^{2},(y z)^{\ell},(z x)^{m}, \ldots\right\rangle
$$

where the dots indicate possible presence of additional relators (at least one if the carrier surface of the map is not simply connected). In particular, all vertices of $\mathcal{M}$ have degree $\ell$ and all the face boundary walks in $\mathcal{M}$ have length $m$; we will often refer just to face length $m$. The pair $(\ell, m)$ is the type of the regular map $\mathcal{M}$. In such a representation of $\mathcal{M}$, its flags are elements of $G$, the darts are (say) right cosets of the subgroup $\langle x\rangle$, while edges, vertices and faces are right cosets of the dihedral subgroups $\langle x, y\rangle,\langle y, z\rangle$ and $\langle z, x\rangle$ of order $4,2 \ell$ and $2 m$, respectively. The three generators $x, y, z$ correspond to involutory automorphisms of $\mathcal{M}$ taking a fixed flag onto its three neighboring flags, and the three dihedral subgroups correspond to the edge-, vertex- and face-stabilizers of $\mathcal{M}$.

We will write $\mathcal{M}=\operatorname{Map}(G ; x, y, z)$ to formally identify a regular map $\mathcal{M}$ with a group presentation as in (1.1). The algebraic situation with finite orientably-regular maps is similar. Each such map $\mathcal{M}$ can be identified with a partial two-generator presentation of a group $H$, isomorphic to the group $\operatorname{Aut}^{+}(\mathcal{M})$ of orientation-preserving automorphisms of $\mathcal{M}$, of the form

$$
H=\left\langle\rho, \lambda \mid \rho^{\ell}, \lambda^{2},(\rho \lambda)^{m}, \ldots\right\rangle \text {. }
$$

Here, elements of $H$ represent darts of $\mathcal{M}$; right cosets of the cyclic groups $\langle\lambda\rangle,\langle\rho\rangle$ and $\langle\rho \lambda\rangle$ represent edges, vertices and faces of $\mathcal{M}$. The generators $\lambda$ and $\rho$, stabilizing an edge $e$ and a vertex $v$ incident to $e$, represent a half-turn of $\mathcal{M}$ about the center of $e$ and a $2 \pi / \ell$ turn of $\mathcal{M}$ about $v$ in accord with a chosen orientation of the carrier surface of the map. Again, the pair $(\ell, m)$ is the type of the map, and we will use the notation $\mathcal{M}=\operatorname{Map}(H ; \rho, \lambda)$ in this case. 
If a regular map $\mathcal{M}=\operatorname{Map}(G ; x, y, z)$ is orientable (meaning that its carrier surface is orientable), $\mathcal{M}$ is also orientably-regular, with $\operatorname{Aut}^{+}(\mathcal{M})=\langle\rho, \lambda\rangle$ for $\lambda=x y$ and $\rho=y z$. In fact, a regular map $\operatorname{Map}(G ; x, y, z)$ is orientable if and only if the subgroup $\langle x y, y z\rangle$ has index 2 in $G$. Reversing this line of thought, an orientably-regular map $\mathcal{M}=\operatorname{Map}(H ; \rho, \lambda)$ may also be regular. It happens if and only if the map admits an orientation-reversing automorphism, which (see e.g. [16]) is equivalent to the existence of an automorphism of $H$ that fixes $\lambda$ and inverts $\rho$. In such a case we call the orientably-regular map $\mathcal{M}$ reflexible; otherwise, that is, when $H \cong \operatorname{Aut}^{+}(\mathcal{M})=\operatorname{Aut}(\mathcal{M})$, the map is called chiral.

A Cayley graph $\operatorname{Cay}(G, X)$ is a graph whose vertex set can be identified with the elements of a group $G$ generated by a set $X$ closed under taking inverses and not containing the identity $1_{G}$, with the pairs of adjacent vertices consisting of all pairs $g, g x$ with $g \in G$ and $x \in X$. A graph $\Gamma$ is isomorphic to a Cayley graph $\operatorname{Cay}(G, X)$ if and only if Aut $\Gamma$ contains a subgroup $G$ acting regularly on the vertices of $\Gamma$ [15]. A Cayley map is an orientable map $\mathcal{M}$ that admits a group of orientation preserving automorphisms $G$ acting regularly on its set of vertices. Therefore, the underlying graphs of Cayley maps are Cayley graphs. It turns out that many of the orientably-regular maps obtained in the forthcoming sections fall in the class of Cayley maps the theory of which (without regularity assumptions) was initiated in [14] and further developed e.g. in [8] and [4].

An orientably-regular Cayley map can therefore be distinguished by $\mathcal{M}=\operatorname{Map}(H$; $\rho, \lambda)$, where $H=J\langle\rho\rangle$ for some subgroup $J \leq H$ such that $J \cap\langle\rho\rangle=1$, vertices of $\mathcal{M}$ are right cosets of $\langle\rho\rangle$ in $H$, and the underlying graph of $\mathcal{M}$ is a Cayley graph $\operatorname{Cay}(J, S)$ for some unit-free inverse-closed generating set $S$ of $J$. In the even more special instance when $J$ is normal in $H$, i.e., when $H$ is a semi-direct product $J \rtimes\langle\rho\rangle$, we speak about a normal (orientably-regular) Cayley map. In this case, conjugation by $\rho$ induces an automorphism $\hat{\rho}$ of $J$ and its restriction $\pi=\pi_{\hat{\rho}}$ to $S$ is a cyclic permutation of $S$. It turns out that either all elements in $S$ are involutions, or none of them is and then $s^{-1}=s \hat{\rho}^{\ell / 2}=\rho^{-\ell / 2} s \rho^{\ell / 2}$ for every $s \in S$, where $\ell$ is the order of $\rho$ (necessarily even in this case). Moreover, since we also know that $J\langle\rho\rangle=\langle\lambda, \rho\rangle$, the involution $\lambda$ can be taken to be equal to an arbitrary element of $S$ in the all-involutions case, or to $s \rho^{\ell / 2}$ for an arbitrary $s \in S$ if no element in $S$ is involutory.

In our paper we address the natural question whether for a given finite Frobenius group $G$ there exists some orientably-regular or even regular map whose automorphism group is $G$. In Section 2, we list some properties of Frobenius groups which we will refer to in Section 3. In Section 3, necessary and sufficient conditions (Theorems 3.3, 3.5 and 3.6) for a Frobenius group to be the automorphism group of an orientably-regular chiral map are given. The Frobenius groups conforming to these conditions are also constructed.

\section{Frobenius groups}

A Frobenius group is a transitive permutation group $G$ on a set $\Omega$ which is not regular on $\Omega$, but has the property that the only element of $G$ which fixes more than one point is the identity element. It was shown by Thompson $[17,18]$ that a finite Frobenius group $G$ has a nilpotent normal subgroup $K$, called the Frobenius kernel, which acts regularly on $\Omega$. Thus, $K$ is the direct product of its Sylow subgroups and $G$ is the semidirect product $K \rtimes H$, where $H$ is the stabilizer of a point of $\Omega$. Because of the vertex transitivity of the action, any two point stabilizers are conjugate. As a result, every point stabilizer has the form $(h k)^{-1} H(h k)=k^{-1} H k=H^{k}$ for some $h \in H$ and $k \in K$. Each point stabilizer is 
called a Frobenius complement of $K$ in $G$, so the choice of Frobenius complement is not unique. Because of the regularity of $K$ acting on $\Omega$, one may identify $\Omega$ with $K$ such that $K$ acts on itself by multiplication. Moreover, Gorenstein [6, pp. 38, 339] showed that every element of $H \backslash\{1\}$ induces an automorphism of $K$ by conjugation which fixes only the identity element of $K$. Combining all these results we give a lemma to express the relation between a Frobenius group and its Frobenius kernel as well as its Frobenius complements.

Lemma 2.1. Let $G=K \rtimes H$ be a Frobenius group, where $K$ is the Frobenius kernel and $H$ is a Frobenius complement. Then, $G$ can be divided in the following two ways.

(1) $G=\cup_{k \in K} H k$, where $H k_{1} \cap H k_{2}=\emptyset$ for any two different elements $k_{1}, k_{2} \in K$;

(2) $G=\left(\cup_{k \in K} H^{k}\right) \cup K$, where $H^{k}=k^{-1} H k$ denotes the conjugation of $H$ by $k$, and $H^{k_{1}} \cap H^{k_{2}}=H^{k} \cap K=\{1\}$ for any elements $k_{1}, k_{2}, k$ in $K$ and $k_{1} \neq k_{2}$.

Given a(several) Frobenius group(s), one can get new Frobenius groups. In the following Lemmas 2.3 and 2.4, we give two methods to get new Frobenius groups from original ones.

Lemma 2.2 ([19, Lemma 3.8, p. 13]). Assume $A, B$ are two groups and $B$ acts on $A$. If $A$ has a subgroup $P$ which is invariant under the action of $B,(|B|,|P|)=1$ and $(P a)^{b}=P a$ for some $a \in A$ and each $b \in B$, then there is an element $x \in P a$ such that $x^{b}=x$ for every $b \in B$.

Lemma 2.3. Assume $G=K \rtimes H, 1<N<K$ and $N \unlhd G$. Then $G$ is a Frobenius group with $H$ as a Frobenius complement if and only if both $N \rtimes H$ and $K / N \rtimes H$ are Frobenius groups with $H$ as a Frobenius complement.

Proof. Assume $G=K \rtimes H$ is a Frobenius group with $H$ as a Frobenius complement. It is obvious that $N \rtimes H$ is a Frobenius group with $H$ a Frobenius complement. So we only need to show that $K / N \rtimes H$ is a Frobenius group. If not, then there is an $h \in H$ such that $h$ fixes some non-identity element of $K / N$. That is, there is an element $k \in K$ but $k \notin N$ such that $N k$ is fixed by $h$. Consider $K \rtimes\langle h\rangle$. From Lemma 2.2, there is an element $x \in N k$ which is fixed by $h$. It is obvious that $x \neq 1$, and so $h$ fixes at least two elements in $K$. This contradicts the assumption of $G$ being a Frobenius group.

Conversely, assume both $N \rtimes H$ and $K / N \rtimes H$ are Frobenius groups with $H$ as a Frobenius complement. If $G$ is not a Frobenius group, then there exists $1 \neq k \in K$ and $1 \neq h \in H$ such that $k^{h}=k$. Since $N \rtimes H$ is a Frobenius group, $k \notin N$. Thus $N k \neq \overline{1}$ in $K / N$. Clearly, $(N k)^{h}=N k^{h}=N k$. This contradicts the assumption of $K / N \rtimes H$ being a Frobenius group.

Lemma 2.4. Let $K_{1} \rtimes H$ and $K_{2} \rtimes H$ be two Frobenius groups. Then, $\left(K_{1} \times K_{2}\right) \rtimes H$ is a Frobenius group, where $H$ acts on $K_{1} \times K_{2}$ by $\left(k_{1} k_{2}\right)^{h}=k_{1}^{h} k_{2}^{h}$, for any elements $k_{1} \in K_{1}, k_{2} \in K_{2}$ and $h \in H$.

Proof. Note that each non-identity element $h \in H$ fixes exactly the identity element of $K_{1} \times K_{2}$.

Lemma 2.5. Let $G=K \rtimes H$ be a Frobenius group. For each $g \in G \backslash K$, it satisfies the following two relations:

(1) $\langle g\rangle \cap K=\{1\}$; 
(2) As an element in the quotient group $G / K, K g$ has order $o(g)$, where $o(g)$ denotes the order of $g$ in group $G$.

Proof. According to Lemma 2.1, there is an element $k \in K$ such that $g \in H^{k}$. So $\langle g\rangle \cap$ $K \leq H^{k} \cap K=\{1\}$. As a result, $o(K g)=|K\langle g\rangle / K|=|\langle g\rangle| /\langle g\rangle \cap K|=|\langle g\rangle \mid=$ $o(g)$.

Corollary 2.6. Let $G=K \rtimes H$ be a Frobenius group. For each $h \in H, h \neq 1$ and for each $k \in K$, the orders of $h, k h$ and $h k$ are equal, that is $o(h)=o(k h)=o(h k)$.

Proof. Note that $K h=K(k h)=K(h k)$, so $o(h)=o(k h)=o(h k)$ according to Lemma 2.5(2).

Remark 2.7. If a group $G=N \rtimes P$ is not a Frobenius group, then it may not satisfy the results in Lemma 2.5. For example, take $G=\mathrm{SL}_{2}(3) \cong Q_{8} \rtimes \mathbb{Z}_{3}$. Let $x=\left(\begin{array}{cc}0 & 1 \\ -1 & 1\end{array}\right)$. There $x \in G \backslash Q_{8}, o(x)=6$. But $\langle x\rangle \cap Q_{8}=\left\langle x^{3}\right\rangle \neq 1$ and $o\left(Q_{8} x\right)=3 \neq o(x)$.

\section{Maps having Frobenius groups as automorphism groups}

The following Lemma 3.1 will be referred to several times in this paper. The result is known and one can prove it very quickly. But for easy reference, we give a short proof.

Lemma 3.1. Let $G$ be a finite group. If there is an involution $\tau \in \operatorname{Aut}(G)$ such that $\tau$ only fixes the identity element of $G$, then $\tau$ maps each element in $G$ to its inverse and $G$ is an abelian group of odd order.

Proof. According to the property of $\tau$, one can check that $G=\left\{g^{-1} g^{\tau} \mid g \in G\right\}$. Clearly $\left(g^{-1} g^{\tau}\right)^{\tau}=\left(g^{-1} g^{\tau}\right)^{-1}$. It follows that $\tau$ maps each element in $G$ to its inverse. So, for any two elements $a, b \in G$, one can get $(a b)^{\tau}=b^{-1} a^{-1}=a^{\tau} b^{\tau}=a^{-1} b^{-1}$. That is to say, $G$ is an abelian group. Since $\tau$ only fixes the identity element of $G$, the group $G$ does not have involutions. Thus, $G$ is of odd order.

If $a \in L$, then we use $\langle a\rangle^{L}$ to denote the group generated by the elements $x^{-1} a x$ for $x \in L$.

Theorem 3.2. Other than dihedral groups of order $2 n$ for any odd integer $n$, Frobenius groups cannot be the automorphism groups of regular maps.

Proof. Let $G=K \rtimes H$ be a Frobenius group. If $G$ can be the automorphism group of a regular map, then $G$ has the following generating relations:

$$
G=\left\langle x, y, z \mid x^{2}, y^{2}, z^{2},(x y)^{2},(y z)^{k},(z x)^{m}, \ldots\right\rangle,
$$

where $2, k, m$ are the true orders of $x y, y z$ and $z x$, respectively. As $G / K=\langle K x, K y$, $K z\rangle \cong H,|H|$ is even. By Lemma $3.1, K$ is an abelian group of odd order and $H$ has a unique involution. Consequently, $H \cong \mathbb{Z}_{2}$. Moreover, $H$ is a Sylow-2 subgroup of $G$.

It is easy to see that $\langle x, y\rangle$ is a 2-group. So $|\langle x, y\rangle| \leq|H|=2$. Thus $\langle x, y\rangle=\langle x y\rangle$. It follows that $x=1$ or $y=1$. In either case, $G$ is a dihedral group of order $2 n$ for some odd integer $n$. In this situation, the map is an embedding of a semi-star of valency $n$ in the sphere or the disc, or the dual of the latter, an embedding of a circuit of length $n$ in the boundary of the disc. It is obvious that these two infinite families of maps are reflexible with 
their full automorphism groups being the dihedral groups of order $2 n$. Apart from these two infinite families of maps, the only other possibilities for Frobenius automorphism groups are orientably-regular chiral maps.

According to Theorem 3.2, we only need to concentrate on Frobenius groups which can be automorphism groups of orientably-regular chiral maps. There are several well-known infinite families of examples of these, such as the embeddings of complete graphs $K_{n}$ [9], Paley graphs, and generalized Paley graphs [10]. In the following Theorem 3.3, we will give the necessary conditions that a Frobenius group $G=K \rtimes H$ should satisfy to be the automorphism group of an orientably-regular chiral map.

Theorem 3.3. Let $G=K \rtimes H$ be a Frobenius group. If $G=\left\langle\rho, \lambda \mid \rho^{k}, \lambda^{2},(\rho \lambda)^{m}, \ldots\right\rangle$, $k, m \geq 3$, is the automorphism group of an orientably-regular chiral map $\mathcal{M}=\operatorname{Map}(G$; $\rho, \lambda)$, then one of the following two cases will happen.

(1) $H$ is a cyclic group of even order and $K$ is an abelian group of odd order. There are two subcases corresponding to the parity of $k$.

(1.1) If $k$ is even, then $H \cong \mathbb{Z}_{k}$ and

$$
m=\left\{\begin{array}{lll}
\frac{k}{2}, & \text { if } k \equiv 2 & (\bmod 4), \\
k, & \text { if } k \equiv 0 & (\bmod 4) .
\end{array}\right.
$$

Moreover, the map $\mathcal{M}$ is an orientably-regular normal Cayley map of $K$. When $k \equiv 2(\bmod 4), \mathcal{M}$ has $\frac{|G|}{k}$ vertices, $\frac{|G|}{2}$ edges, $\frac{2|G|}{k}$ faces and the genus of the corresponding orientable surface is $1-\frac{|G|(6-k)}{4 k} ;$ when $k \equiv 0(\bmod 4)$, $\mathcal{M}$ has $\frac{|G|}{k}$ vertices, $\frac{|G|}{2}$ edges, $\frac{|G|}{k}$ faces and the genus of the corresponding orientable surface is $1-\frac{|G|(4-k)}{4 k}$.

(1.2) If $k$ is odd, then $H \cong \mathbb{Z}_{2 k}$ and $m=2 k$. The map $\mathcal{M}$ is an orientably-regular normal Cayley map of a group isomorphic to $K \rtimes \mathbb{Z}_{2}$. In this situation, $\mathcal{M}$ has $\frac{|G|}{k}$ vertices, $\frac{|G|}{2}$ edges, $\frac{|G|}{2 k}$ faces, so the genus of the corresponding orientable surface is $1-\frac{|G|(3-k)}{4 k}$.

(2) $H$ is a cyclic group of odd order and $H \cong \mathbb{Z}_{k}, K$ is a 2-group and $m=k$. In this situation, $\mathcal{M}$ is an orientably-regular normal Cayley map of $K$. The map $\mathcal{M}$ has $\frac{|G|}{k}$ vertices, $\frac{|G|}{2}$ edges, $\frac{|G|}{k}$ faces and the genus of the corresponding orientable surface is $1-\frac{|G|(4-k)}{4 k}$.

Proof. (1): If $|H|$ is even, then there is an involution in $\operatorname{Aut}(K)$ which only fixes the identity element. So, $K$ is an abelian group of odd order by Lemma 3.1. In this case, $\lambda \notin K$ and so $o(K \lambda)=2$ in the quotient group $G / K$. Note that $\rho \notin K$. Otherwise, by Corollary 2.6 one can get $o(\rho \lambda)=o(\lambda)=2$, that is $m=2$. So, $o(K \rho)=o(\rho)=k$ by Lemma 2.5. According to Lemma 3.1, there is only one involution in $H \cong G / K=$ $\langle K \rho, K \lambda\rangle$, so $K \lambda$ belongs to the center of $G / K$ which is therefore abelian.

(1.1): If $k$ is even, then $K \lambda \in\langle K \rho\rangle$. So, $\langle K \rho, K \lambda\rangle=\langle K \rho\rangle$ and $H \cong \mathbb{Z}_{k}$. According to Lemma 2.1, one can assume $H=\langle\rho\rangle$ and $\lambda=a \rho^{\frac{k}{2}}$ for some non-identity element $a \in K$ without loss of generality. The vertices of $\mathcal{M}$ can be looked as the cosets of $H$. 
Therefore, $K$ acts regularly on the vertices of $\mathcal{M}$ which implies that $\mathcal{M}$ is an orientablyregular Cayley map of $K$. Now, we know that $\operatorname{Aut}(\mathcal{M})=K \rtimes\langle\rho\rangle$. So, $\mathcal{M}$ is normal and from the construction method of $\mathcal{M}$ from $G$, one can get the corresponding Cayley subset $\left\{a, a^{\rho}, a^{\rho^{2}}, \ldots, a^{\rho^{k-1}}\right\}$. In this case, $K=\langle a\rangle^{H}$.

Since $K \lambda=K \rho^{\frac{k}{2}}, K \rho K \lambda=K \rho^{\frac{k}{2}+1}$. If $k \equiv 2(\bmod 4)$, then $m=o(\rho \lambda)=$ $o(K \rho K \lambda)=o\left(K \rho^{\frac{k}{2}+1}\right)=\frac{k}{2}$. The type of $\mathcal{M}$ is $\left(k, \frac{k}{2}\right)$. Moreover, $\mathcal{M}$ has $\frac{|G|}{k}$ vertices, $\frac{|G|}{2}$ edges, $\frac{2|G|}{k}$ faces and the genus of the corresponding orientable surface is $1-\frac{|G|(6-k)}{4 k}$. If $k \equiv 0(\bmod 4)$, then $m=k$ and consequently the type of $\mathcal{M}$ is $(k, k)$. And $\mathcal{M}$ has $\frac{|G|}{k}$ vertices, $\frac{|G|}{2}$ edges, $\frac{|G|}{k}$ faces and the genus of the corresponding orientable surface is $1-\frac{|G|(4-k)}{4 k}$.

(1.2): If $k$ is odd, then $K \lambda$ belongs to the center of $G / K$ which is therefore abelian. So, $\langle K \rho, K \lambda\rangle=\langle K \rho K \lambda\rangle$ and as a result $H \cong \mathbb{Z}_{2 k}$. Because $\rho \notin K$, according to Lemma 2.1, we may assume $\rho \in H$ and $H=\langle\tilde{\rho}\rangle$ with $\rho=\tilde{\rho}^{2}$. As a result, $\lambda=a \tilde{\rho}^{k}$ for some nonidentity element $a \in K$. Because $\lambda \rho=a \tilde{\rho}^{k+2}$, it follows that $m=o(\lambda \rho)=o\left(\tilde{\rho}^{k+2}\right)=2 k$ according to Lemma 2.6. The type of $\mathcal{M}$ in this subcase is therefore $(k, 2 k)$.

Let $\tilde{H}=\langle\rho\rangle$ be the index two subgroup of $H$ and $\tilde{K}=K \rtimes\left\langle\tilde{\rho}^{k}\right\rangle \cong K \rtimes \mathbb{Z}_{2}$. It is clear that $G=\tilde{K} \rtimes \tilde{H}$. Now, $\lambda \in \tilde{K}$ and so we have the relations $G=\langle\rho, \lambda\rangle \leq\langle\lambda\rangle^{\langle\rho\rangle}\langle\rho\rangle \leq$ $\tilde{K} \tilde{H}=G$. Therefore, $\tilde{K}=\langle\lambda\rangle^{\tilde{H}}$.

The vertices of $\mathcal{M}$ can be looked as the cosets of $\tilde{H}$. Therefore, $\tilde{K}$ acts regularly on the vertices of $\mathcal{M}$ which implies that $\mathcal{M}$ is an orientably-regular normal Cayley map of $\tilde{K}$ with corresponding Cayley subset $\left\{\lambda, \lambda^{\rho}, \lambda^{\rho^{2}}, \ldots, \lambda^{\rho^{k-1}}\right\}$. In this case, $\mathcal{M}$ has $\frac{|G|}{k}$ vertices, $\frac{|G|}{2}$ edges, $\frac{|G|}{2 k}$ faces, so the genus of the corresponding orientable surface is $1-\frac{|G|(3-k)}{4 k}$.

(2): If $|H|$ is odd, then $\lambda \in K$ and so $H \cong G / K=\langle K \rho\rangle$ is cyclic. Similar to (1.1), we can assume $H=\langle\rho\rangle$ and $\mathcal{M}$ is an orientalby-regular normal Cayley map of $K$ with the corresponding Cayley subset $\left\{\lambda, \lambda^{\rho}, \ldots, \lambda^{\rho^{k-1}}\right\}$. Also in this case $K=\langle\lambda\rangle^{H}$. It is known that $K$ is nilpotent, so the Sylow-2 subgroup $P$ of $K$ is a characteristic subgroup of $G$. Note that $G=\langle\lambda, \rho\rangle=\langle\lambda\rangle^{G}\langle\rho\rangle \leq P \rtimes\langle\rho\rangle \leq K \rtimes\langle\rho\rangle$. So, $K=\langle\lambda\rangle^{G}=P$ is a 2-group. According to Corollary 2.6, $o(\rho \lambda)=o(\rho)=k$ and so the type of $\mathcal{M}$ is $(k, k)$. The map $\mathcal{M}$ has $\frac{|G|}{k}$ vertices, $\frac{|G|}{2}$ edges, $\frac{|G|}{k}$ faces and the genus of the corresponding orientable surface is $1-\frac{|G|(4-k)}{4 k}$.

In the proof of Theorem 3.3, for a Frobenius group $G=K \rtimes H$ that can be the automorphism group of an orientably-regular chiral map, we have described the relations between $K$ and $H$. To be more clear, we rewrite these relations in Corollary 3.4.

Corollary 3.4. Let $G=K \rtimes H$ be a Frobenius group. If $G=\left\langle\rho, \lambda \mid \rho^{k}, \lambda^{2},(\rho \lambda)^{m}, \ldots\right\rangle$ is the automorphism group of an orientably-regular chiral map $\operatorname{Map}(G ; \rho, \lambda)$, then one of the following three cases will happen:

(1) $k$ is even, $H=\langle\rho\rangle \cong \mathbb{Z}_{k}$, $K$ is an abelian group and $K=\left\langle\lambda \rho^{\frac{k}{2}}\right\rangle^{H}$;

(2) $k$ is odd, $H \cong \mathbb{Z}_{2 k}$, $K$ is abelian and $G=\tilde{K} \rtimes \tilde{H}$, where $\tilde{K} \cong K \rtimes \mathbb{Z}_{2}, \tilde{H}=\langle\rho\rangle$ is the index two subgroup of $H$ and $\tilde{K}=\langle\lambda\rangle^{\tilde{H}}$;

(3) $k$ is odd, $H=\langle\rho\rangle \cong \mathbb{Z}_{k}$ and $K=\langle\lambda\rangle^{H}$ is a 2-group.

In the following Theorems 3.5 and 3.6, we will show that a Frobenius group whose Frobenius kernel and Frobenius complement conforming to the conditions in Corollary 3.4 
can be the automorphism group of an orientably-regular normal Cayley map which implies that the conditions are also sufficient.

Theorem 3.5. Let $G=K \rtimes H$ be a Frobenius group, where $K$ is abelian and $K=\langle x\rangle^{H}$ for some $x \in K, H=\langle y\rangle$ is cyclic of order $2 n, n \geq 2$. Then, there is an orientably-regular normal Cayley map $\mathcal{M}$ such that $G=\operatorname{Aut}(\mathcal{M})$ and the type of $\mathcal{M}$ is

$$
(k, m)= \begin{cases}(2 n, n) \text { or }(n, 2 n), & \text { if } n \text { is odd } \\ (2 n, 2 n), & \text { if } n \text { is even }\end{cases}
$$

Proof. Let $\rho=y, \lambda=x y^{n}$. Then $G=\langle\rho, \lambda\rangle$. It is clear that $o(\rho)=2 n, o(\lambda)=2$, $o(\rho \lambda)=n$ if $n$ is odd and $o(\rho \lambda)=2 n$ if $n$ is even. So, $G$ is the automorphism group of an orientably-regular map $\mathcal{M}$ of type $(2 n, n)$ or $(2 n, 2 n)$ depending on whether $n$ is odd or even. Because $H=\langle y\rangle=\langle\rho\rangle$, it follows that the vertex set consists of the cosets of $H$ in $G$. So, $K$ acts regularly on the vertex set of $\mathcal{M}$ and as a result $\mathcal{M}$ is an orientably-regular normal Cayley map of $K$.

When $n$ is odd, if we set $\rho=y^{2}, \lambda=x y^{n}$, then $o(\rho)=n, o(\lambda)=2$ and $o(\rho \lambda)=2 n$. We claim that $G=\langle\rho, \lambda\rangle$. Set $Q=\langle\rho, \lambda\rangle$, then $Q=\left\langle y^{2}, x y\right\rangle$ because $n$ is odd. From the requirement of $n \geq 2$, we have $y^{2} \neq 1$ and so $C_{K}\left(y^{2}\right)=1$ in the Frobenius group $G$. A calculation shows that the commutator $\left[y^{2}, x^{-1}\right]=(x y)^{y^{2}}(x y)^{-1} \in Q$. Also, $\left[y^{2}, x^{-1}\right]$ belongs to $K$. Note that $K$ is abelian. We have

$$
\begin{aligned}
Q \geq\left[y^{2}, x^{-1}\right]^{\langle x y\rangle} & =\left[y^{2}, x^{-1}\right]^{K\langle x y\rangle}=\left[y^{2}, x^{-1}\right]^{K\langle y\rangle} \\
& =\left[y^{2}, x^{-1}\right]^{\langle y\rangle}=\left\langle\left[y^{2},\left(x^{-1}\right)^{g}\right] \mid g \in\langle y\rangle\right\rangle .
\end{aligned}
$$

Define a function $\sigma: K \rightarrow K$ such that $b^{\sigma}=\left[y^{2}, b\right]$ for each $b \in K$. Now,

$$
\left(b_{1} b_{2}\right)^{\sigma}=\left[y^{2}, b_{1} b_{2}\right]=\left[y^{2}, b_{2}\right]\left[y^{2}, b_{1}\right]^{b_{2}}=\left[y^{2}, b_{1}\right]\left[y^{2}, b_{2}\right]=b_{1}^{\sigma} b_{2}^{\sigma} .
$$

From $C_{K}\left(y^{2}\right)=1$, one can get $\sigma \in \operatorname{Aut}(K)$. Therefore,

$$
\left\langle\left[y^{2},\left(x^{-1}\right)^{g}\right] \mid g \in\langle y\rangle\right\rangle=\left\langle\left(\left(x^{-1}\right)^{g}\right)^{\sigma} \mid g \in\langle y\rangle\right\rangle=\left(\left\langle x^{-1}\right\rangle^{\langle y\rangle}\right)^{\sigma}=K^{\sigma}=K .
$$

So, $K \leq Q$ and $\langle x y\rangle K \leq Q$. Consequently, $Q=G$. Let $\tilde{K}=K \rtimes\left\langle y^{n}\right\rangle$ and $\tilde{H}=\langle\rho\rangle$. Then, $\tilde{\tilde{K}} \cong K \rtimes \mathbb{Z}_{2}, \tilde{H}$ is the index two subgroup of $H$ and $G=\tilde{K} \rtimes \tilde{H}$. Therefore, $G$ is the automorphism group of an orientably-regular normal Cayley map of $\tilde{K}$ of type $(n, 2 n)$.

Theorem 3.6. Let $G=K \rtimes H$ be a Frobenius group, where $K$ is a 2-group and $K=\langle x\rangle^{H}$ for some involution $x \in K, H=\langle y\rangle$ is cyclic of order $n$ for some odd integer $n$. Then, there is an orientably-regular normal Cayley map $\mathcal{M}$ such that $G=\operatorname{Aut}(\mathcal{M})$ and the type of $\mathcal{M}$ is $(n, n)$.

Proof. Let $\rho=y, \lambda=x$. Then $G=\langle\rho, \lambda\rangle$. It is clear that $o(\rho)=n, o(\lambda)=2, o(\rho \lambda)=n$. So, $G$ is the automorphism group of an orientably-regular map $\mathcal{M}$ of type $(n, n)$. Because $H=\langle y\rangle=\langle\rho\rangle$, it follows that the vertex set consists of the cosets of $H$ in $G$. So, $K$ acts regularly on the vertex set of $\mathcal{M}$ and as a result $\mathcal{M}$ is an orientably-regular normal Cayley map of $K$. 
Corollary 3.7. Let $K_{1} \rtimes H$ and $K_{2} \rtimes H$ be Frobenius groups, where $K_{1}=\left\langle x_{1}\right\rangle^{H}$, $K_{2}=\left\langle x_{2}\right\rangle^{H}$ are both abelian groups whose orders are coprime with each other, $H \cong\langle y\rangle$ and $o(y)=2 n$ for some integer $n \geq 2$. Then, the following two results follow from Lemma 2.4 and Theorem 3.5.

- $\left(K_{1} \times K_{2}\right) \rtimes H$ is a Frobenius group, $K_{1} \times K_{2}=\left\langle x_{1} x_{2}\right\rangle^{H}$ and for each $a_{1} \in$ $K_{1}, a_{2} \in K_{2}, b \in H$ the element $b$ acts on $a_{1} a_{2}$ in the way $\left(a_{1} a_{2}\right)^{b}=a_{1}^{b} a_{2}^{b}$,

- $\left(K_{1} \times K_{2}\right) \rtimes H$ is the automorphism group of an orientably-regular normal Cayley map.

According to Theorem 3.5 and Corollary 3.7, one may concentrate on Frobenius groups whose Frobenius kernels are $p$-groups and satisfy the conditions in Theorem 3.5. Now, we want to give an example of Frobenius groups satisfying the conditions in Theorem 3.5.

In a finite group $G$, for each element $g \in G$ we use $C_{G}(g)$ to denote the centralizer of $g$ in $G$, that is $C_{G}(g)=\{h \in G \mid h g=g h\}$.

Example 3.8. Let $K=\left\langle a_{1}\right\rangle \times\left\langle a_{2}\right\rangle \times \cdots \times\left\langle a_{d}\right\rangle$, where $o\left(a_{i}\right)=p^{e_{i}}, p$ is an odd prime number and these positive integers $e_{i}, 1 \leq i \leq d$, satisfy $e_{1} \geq e_{2} \geq \cdots \geq e_{d}$. Let $H \cong \mathbb{Z}_{k}=\langle b\rangle$ for some positive even integer $k$ satisfying $k \mid p-1$. Assume $d \leq \phi(k)$, where $\phi$ is the Euler's totient function, $t_{i}$ is a positive integer such that $t_{i}+p^{e_{i}} \mathbb{Z}$ is an element in $\mathbb{Z}_{p^{e_{i}}}^{*}$ of order $k$ and $t_{i}+p \mathbb{Z} \neq t_{j}+p \mathbb{Z}$ for any $1 \leq i \neq j \leq d$. Set $G=K \rtimes H$, where $a_{i}^{b}=a_{i}^{t_{i}}$, then $G$ is a Frobenius group. Take $a=\prod_{i=1}^{d} a_{i}$, then

$$
K=\langle a\rangle^{H}=\left\langle a, a^{b}, \ldots, a^{b^{d-1}}\right\rangle .
$$

Proof. To show that $G$ is a Frobenius group, we only need to show that for each element $y \in H \backslash\{1\}$, the equality $C_{K}(y)=1$ holds. Suppose $x=\prod_{i=1}^{d} x_{i} \in C_{K}(y)$, where $x_{i} \in\left\langle a_{i}\right\rangle$. It is obvious that $\prod_{i=1}^{d} x_{i}=\prod_{i=1}^{d} x_{i}^{y}$. From the defining relation $a_{i}^{b}=a_{i}^{t_{i}}$, then $\left\langle a_{i}\right\rangle$ is an $H$-invariant subgroup, and so $x_{i}=x_{i}^{y}$ for each $i$. That is $x_{i} \in C_{\left\langle a_{i}\right\rangle}(y)$. While $y$ is a power of $b$ and the action of $b$ on $\left\langle a_{i}\right\rangle$ has only one fixed point, that is the identity of $\left\langle a_{i}\right\rangle$, so $C_{\left\langle a_{i}\right\rangle}(y)=1$.

For each $1 \leq \ell \leq d-1, a^{b^{\ell}}=\prod_{i=1}^{d} a_{i}^{t_{i}^{\ell}}$. If we look at the determinant

$$
\left|\begin{array}{cccc}
1 & 1 & \cdots & 1 \\
t_{1} & t_{2} & \cdots & t_{d} \\
\vdots & \vdots & \vdots & \vdots \\
t_{1}^{d-1} & t_{2}^{d-1} & \cdots & t_{d}^{d-1}
\end{array}\right|
$$

in the finite field $F_{p}$, then from the choices of $t_{i}$ this is a non-zero Vandermonde determinant. As a result,

$$
a+\Phi(K), a^{b}+\Phi(K), \ldots, a^{b^{d-1}}+\Phi(K)
$$

is a basis of the linear space $K / \Phi(K)$, where $\Phi(K)$ is the Frattini subgroup of $K$. From the Burnside basis theorem, the result $K=\left\langle a, a^{b}, \ldots, a^{b^{d-1}}\right\rangle$ follows.

Corollary 3.9. Let $K$ and $H$ be groups in Example 3.8. Then, the Frobenius group $G=$ $K \rtimes H$ is the automorphism group of an orientably-regular normal Cayley map described in Theorem 3.5. 
Lemma 3.10. Let $A$ be a group, $B$ be a subgroup of $A$ of index 3 , and each $a \in A \backslash B, a^{3}=$ 1. Then, $\left[b, b^{a}\right]=1$ for any $b \in B, a \in A \backslash B$.

Proof. Note that if $b \in B$ and $a \in A \backslash B$, then $b a \in A \backslash B$. So, $(b a)^{3}=1$ and $b a b=a^{-1} b^{-1} a^{-1}$. The commutator $\left[b, b^{a}\right]=b^{-1} a^{-1} b^{-1} a b a^{-1} b a=b^{-1}\left(a^{-1} b^{-1} a^{-1}\right)$. $\left(a^{-1} b a^{-1}\right) b a=b^{-1}(b a b)\left(b^{-1} a b^{-1}\right) b a=a^{3}=1$.

Corollary 3.11. Let $G=K \rtimes H$ be a Frobenius group. If $G$ satisfies the following two conditions:

(1) G can be generated by two elements,

(2) $H \cong \mathbb{Z}_{3}$,

then $K$ is abelian. Moreover, if $G$ is the automorphism group of an orientably-regular map, then $K$ is isomorphic to the Klein group $K_{4}$ and $G$ is isomorphic to the alternating group $A_{4}$.

Proof. Assume $G=\langle a, b\rangle$ and $a \notin K$. By Lemma 2.1 and Corollary 2.6, $a^{3}=1$ and so $G=K \cup K a \cup K a^{2}$. As a result, one of the three elements $b, b a^{-1}, b a^{-2}$ must belong to $K$. Suppose $b \in K$, then $G=\langle a, b\rangle=\langle a\rangle\langle b\rangle^{G} \leq\langle a\rangle K=G$. Because $\langle a\rangle \cap K=1$, it follows that $K=\langle b\rangle^{G}$. While $\langle b\rangle^{G}=\left\langle b, b^{a}, b^{a^{2}}\right\rangle$, so $K$ is abelian according to Lemma 3.10.

If $G$ is the automorphism group of an orientably-regular map, then without loss of generality we can assume $H \cong\langle a\rangle$. So, $K=\left\langle b, b^{a}, b^{a^{2}}\right\rangle$ is a 2-group according to Theorem 3.3. The fact of $K$ being abelian implies that the rank $d(K)$ of $K$ satisfies $d(K) \leq 3$. Therefore, $K \cong \mathbb{Z}_{2}^{d(K)}$. Moreover, from 3||$K \mid-1$, one can get $d(K)=2$ and $K$ is isomorphic to $K_{4}$ and $G \cong A_{4}$.

Remark 3.12. In Corollary 3.11, the condition $K=\langle b\rangle^{H}$ for some element $b \in K$ is necessary. In fact, one may check the list of small groups to find $\operatorname{SmallGroup}(192,1023)$ in MAGMA [1] to get a Frobenius group satisfying $K=\langle a, b\rangle^{H}$ for two different elements $a$ and $b$ of $K, H \cong \mathbb{Z}_{3}$, but $K$ is not abelian.

According to Theorem 3.3, if the Frobenius group $G=K \rtimes H$ is the automorphism group of an orientably-regular map and $|H|$ is odd, then $K$ is a 2-group. By Corollary 3.11, in order to find a non-abelian 2-group as the Frobenius kernel, the smallest order of the Frobenius complement is 5 .

Theorem 3.13. Let $G=K \rtimes H$ be a Frobenius group. If $K$ is a non-abelian 2-group, $H$ is a cyclic group of odd order and $G$ is the automorphism group of an orientably-regular map, then the group $G$ of the smallest order is SmallGroup $(1280,1116310)$ in MAGMA.

Proof. Since $K$ is a non-abelian 2-group, its commutator subgroup $K^{\prime}$ is non-trivial and is a proper subgroup of $K$. Set $|H|=n$. Because $K / K^{\prime} \rtimes H$ and $K^{\prime} \rtimes H$ are both Frobenius groups, $n \mid\left(\left|K / K^{\prime}\right|-1\right)=2^{n_{1}}-1$ and $n \mid\left(\left|K^{\prime}\right|-1\right)=2^{n_{2}}-1$ for some integers $n_{1}$ and $n_{2}$. According to Corollary 3.11, $n$ is an odd integer but $n \neq 3$.

If $n=5$, then the smallest choices of $n_{1}, n_{2}$ are 4 and in this case $|G|=2^{8} \times 5$. The Frobenius group satisfying these conditions really exists. It is $\operatorname{SmallGroup}(1280,1116310)$ in the list of groups in MAGMA.

We claim that no Frobenius groups of order less than $2^{8} \times 5$ with non-abelian 2-groups as Frobenius kernels, cyclic groups of odd orders as Frobenius complements, exist that can 
be automorphism groups of orientably-regular maps. Otherwise, suppose a group $G=$ $\left\langle\rho, \lambda \mid \rho^{n}, \lambda^{2}, \ldots\right\rangle$ satisfies these conditions. Then, $n=7, n_{1}=n_{2}=3$ and $|G|=$ $2^{6} \times 7=448$. It is $\operatorname{SmallGroup}(448,1394)$ in the list of groups of MAGMA. But, its Frobenius kernel is abelian which is a contradiction.

\section{ORCID iDs}

Haipeng Qu (D) https://orcid.org/0000-0002-3858-5767

Yan Wang (D) https://orcid.org/0000-0002-0148-2932

Kai Yuan (D) https://orcid.org/0000-0003-1858-3083

\section{References}

[1] W. Bosma, J. Cannon and C. Playoust, The Magma algebra system I: The user language, J. Symbolic Comput. 24 (1997), 235-265, doi:10.1006/jsco.1996.0125.

[2] R. P. Bryant and D. Singerman, Foundations of the theory of maps on surfaces with boundary, Quart. J. Math. Oxford 36 (1985), 17-41, doi:10.1093/qmath/36.1.17.

[3] W. Burnside, Theory of Groups of Finite Order, Cambridge University Press, 2nd edition, 1911, doi:10.1017/cbo9781139237253.

[4] M. Conder, R. Jajcay and T. Tucker, Regular Cayley maps for finite abelian groups, J. Algebraic Combin. 25 (2007), 259-283, doi:10.1007/s10801-006-0037-0.

[5] W. Dyck, Ueber Aufstellung und Untersuchung von Gruppe und Irrationalität regulärer Riemann'scher Flächen, Math. Ann. 17 (1880), 473-509, doi:10.1007/bf01446929.

[6] D. Gorenstein, Finite Groups, Harper \& Row, New York-London, 1968.

[7] L. Heffter, Ueber metacyklische Gruppen und Nachbarconfigurationen, Math. Ann. 50 (1898), 261-268, doi:10.1007/bf01448067.

[8] R. Jajcay and J. Širáň, Skew-morphisms of regular Cayley maps, Discrete Math. 244 (2002), 167-179, doi:10.1016/s0012-365x(01)00081-4.

[9] L. D. James and G. A. Jones, Regular orientable imbeddings of complete graphs, J. Comb. Theory Ser. B 39 (1985), 353-367, doi:10.1016/0095-8956(85)90060-7.

[10] G. A. Jones, Characterisations and Galois conjugacy of generalised Paley maps, J. Comb. Theory Ser. B 103 (2013), 209-219, doi:10.1016/j.jctb.2012.07.006.

[11] G. A. Jones and D. Singerman, Theory of maps on orientable surfaces, Proc. London Math. Soc. 37 (1978), 273-307, doi:10.1112/plms/s3-37.2.273.

[12] J. Kepler, The Harmony of the World, volume 209 of Memoirs of the American Philosophical Society, American Philosophical Society, Philadelphia, PA, 1997.

[13] F. Klein, Ueber die Transformation siebenter Ordnung der elliptischen Functionen, Math. Ann. 14 (1878), 428-471, doi:10.1007/bf01677143.

[14] R. B. Richter, J. Širáň, R. Jajcay, T. W. Tucker and M. E. Watkins, Cayley maps, J. Comb. Theory Ser. B 95 (2005), 189-245, doi:10.1016/j.jctb.2005.04.007.

[15] G. Sabidussi, Vertex-transitive graphs, Monatsh. Math. 68 (1964), 426-438, doi:10.1007/ bf01304186.

[16] J. Širáň, How symmetric can maps on surfaces be?, in: S. R. Blackburn, S. Gerke and M. Wildon (eds.), Surveys in Combinatorics 2013, Cambridge University Press, Cambridge, volume 409 of London Mathematical Society Lecture Note Series, pp. 161-238, 2013, doi: 10.1017/cbo9781139506748.006, papers from the 24th British Combinatorial Conference held in Egham, July 2013. 
[17] J. Thompson, Finite groups with fixed-point-free automorphisms of prime order, Proc. Nat. Acad. Sci. U.S.A. 45 (1959), 578-581, doi:10.1073/pnas.45.4.578.

[18] J. G. Thompson, Normal p-complements for finite groups, Math. Z. 72 (1960), 332-354, doi: 10.1007/bf01162958.

[19] M. Y. Xu, J. H. Huang, H. L. Li and S. R. Li, Introduction to Group Theory (in Chinese), Science Press, 1999. 